INTERSTITIAL LUNG DISEASE

\title{
Circulating KL-6 levels in patients with drug induced pneumonitis
}

\author{
H Ohnishi, A Yokoyama, Y Yasuhara, A Watanabe, T Naka, H Hamada, M Abe, K Nishimura, \\ J Higaki, J lkezoe, N Kohno
}

Thorax 2003;58:872-875

See end of article for authors' affiliations

....................

Correspondence to: $\operatorname{Dr}$ A Yokoyama, Second Department of Internal Medicine, Ehime University School of Medicine,

Onsen-gun, Ehime 791-

0295, Japan;

yokoyan@m.ehime-u.ac.jp

Revised version received 25 November 2002

Accepted for publication 22 June 2003

Background: The circulating level of $\mathrm{KL}-6 / \mathrm{MUCl}$ is a sensitive marker for various interstitial lung diseases. Previous case reports have suggested that KL- 6 may also be increased in some patients with drug induced pneumonitis. A study was undertaken to determine whether serum KL-6 could be a marker for particular types of drug induced pneumonitis.

Methods: The findings of high resolution computed tomographic (HRCT) chest scans of 30 patients with drug induced pneumonitis were reviewed separately by two independent observers. The pneumonitis was classified into four predominant patterns: widespread bilateral consolidation (diffuse alveolar damage, $D A D ; n=7$ ), fibrosis with or without consolidation (chronic interstitial pneumonia, $C I P ; n=11$ ), consolidation without fibrosis (bronchiolitis obliterans organising pneumonia or eosinophilic pneumonia, $\mathrm{BOOP} / \mathrm{EP} ; \mathrm{n}=8$ ), and diffuse ground glass opacities without fibrosis (hypersensitivity pneumonitis, HP; $\mathrm{n}=4$ ). Serum KL-6 levels were measured by a sandwich enzyme linked immunosorbent assay.

Results: The overall sensitivity of serum KL-6 in detecting drug induced lung disease was $53.3 \%$, which was lower than its sensitivity in detecting other interstitial lung diseases. However, the KL-6 level was increased in most patients with a DAD or CIP pattern $(16 / 18 ; 88.9 \%)$ and was closely correlated with their clinical course. In contrast, serum KL-6 levels were within the normal range in all patients with a BOOP/EP or HP pattern.

Conclusions: Particular patterns detected by HRCT scanning, such as DAD and CIP but not the BOOP/EP or HP patterns, are associated with increased circulating KL-6 levels in drug induced pneumonitis. Serum $\mathrm{KL}-6$ levels may reflect the clinical activity of the particular disorders.

$\mathrm{N}$ umerous agents including cytotoxic and non-cytotoxic drugs have the potential to cause pulmonary toxicity. ${ }^{1-6}$ The clinical and radiological manifestations of drug inducedlungdiseasegenerallyreflecttheunderlyingpathological reactions, which may include interstitial pneumonitis, pulmonary haemorrhage, pulmonary hypertension, non-cardiogenic pulmonaryoedema, veno-occlusivedisease, and granulomatous inflammation. ${ }^{1-7}$ Interstitial pneumonitis can be further classified into four predominant categories: diffuse alveolar damage (DAD), chronic interstitial pneumonia (CIP), bronchiolitis obliterans organising pneumonia or eosinophilic pneumonia (BOOP/EP), and hypersensitivity pneumonitis (HP). The patterns on the high resolution computed tomographic (HRCT) scan of the chest may reflect these four primary categories of the disease. ${ }^{8-10}$ Early diagnosis is important, since withdrawal of the drug responsible is usually sufficient treatment for most drug toxicities, whereas unrecognised toxicity can be progressive and even fatal. However, there is no specific hallmark for diagnosis so the recognition of drug induced disease is difficult. The clinical, radiographic, and histopathological findings are often nonspecific. Affected patients frequently have complex underlying illness, and the use of multiple drugs is common. ${ }^{1-6}$ The diagnosis is based on a history of drug exposure, histopathological evidence of lung damage, and exclusion of other causes of lung injury.

KL-6 is a high molecular weight, mucin like glycoprotein classified as human MUCl mucin ${ }^{11}{ }^{12}$ which has been reported to serve as a sensitive marker for interstitial lung diseases such as idiopathic pulmonary fibrosis, collagen vascular disease associated interstitial pneumonitis, radiation pneumonitis, hypersensitivity pneumonitis, pulmonary sarcoidosis, pulmonary alveolar proteinosis, and Pneumocystis carinii pneumonia. ${ }^{13-20}$ There are a few case reports of increased serum levels of KL-6 in drug induced lung disease, including those associated with herbal Shosaiko-to, amiodarone, misoprostol, and methotrexate. ${ }^{21-24}$ However, the sensitivity and clinical significance of serum KL-6 levels in drug induced lung disease have not been studied. Moreover, there have been no studies of the relationship between the type or extent of damage detected by chest HRCT findings and serum KL- 6 levels. This study was undertaken to clarify these points.

\section{METHODS}

\section{Subjects}

The study population consisted of 30 patients ( 16 men) with drug induced pneumonitis occurring during the period between 1 January 1992 and 31 March 2002. The diagnoses were based on history of drug exposure, pathological evidence of lung damage, and exclusion of other causes of lung injury. The evidence for lung damage and exclusion of other causes were obtained by HRCT scanning and other methods including bronchoalveolar lavage $(n=21)$, transbronchial lung biopsy $(n=17)$, and necropsy $(n=2)$. The causative drugs were determined by history of drug exposure and response to withdrawal of the implicated drug, in addition to a lymphocyte stimulation test $(\mathrm{n}=24)$, skin test $(n=1)$, or incidental provocative drug challenge test $(n=6)$. All of the patients were identified as definite cases on the adverse drug reaction probability scale, ${ }^{25}$ and there was a lack of other explanations for their new lung lesions. The median age of the patients was 62 years (range 23-82). Informed consent was obtained from all patients. 


\section{Chest HRCT scanning and interpretation of HRCT images}

All patients underwent chest radiography and CT scanning. The maximum time interval between the chest CT scan and the serum collection was 4 days, with most examinations $(23 / 30)$ being performed on the same day. After standard $10 \mathrm{~mm}$ thick helical scanning for the screening of chest abnormalities, the HRCT scan was performed with $1-2 \mathrm{~mm}$ collimation at $2-3 \mathrm{~cm}$ intervals from the apex to the base.

The chest radiographic, CT, and HRCT findings were reviewed separately in random order by two independent observers who were not aware of the patients' profiles, and were grouped into four predominant patterns according to previously reported procedures. ${ }^{8-10}$ These included (1) a DAD pattern characterised by widespread bilateral consolidation, (2) a CIP pattern characterised by fibrosis and/or consolidation, (3) a BOOP/EP pattern showing peribronchial or subpleural consolidation without fibrosis, and (4) an HP pattern with diffuse ground glass opacities without fibrosis. A fibrosis was defined by irregular linear opacities with architectural distortion. Ground glass opacities were defined as a diffuse increase in lung density that did not obscure the pulmonary vasculature. The diagnosis of the four predominant patterns seen on the chest HRCT scans was supported by histopathological evidence from bronchoalveolar lavage, transbronchial lung biopsy, and/or necropsy. The extent of the opacities seen on the chest HRCT scans was evaluated using a visual estimating score whereby calculations were made to the nearest $10 \%$ of parenchymal involvement of the whole lung. Following the initial independent evaluations, divergent observations were resolved by consensus after consultation between the two observers.

\section{Measurement of serum KL-6 levels}

Serum samples were collected from all patients at the time of their diagnosis; $2-12$ serum samples per patient were also obtained weekly during the clinical course for 26 patients. Serum KL-6 was measured by a sandwich enzyme linked immunosorbent assay using the KL-6 antibody as described previously. ${ }^{11}$ The cut off value for serum KL-6 was set at $520 \mathrm{U} / \mathrm{ml}$ according to a previous report. ${ }^{13}$

\section{Statistical analysis}

The interobserver agreement was assessed using Kappa statistics. ${ }^{26}$ Kappa values greater than 0.61 were considered as indicating good agreement between observers. ${ }^{26}$ The serum KL-6 levels in the four chest HRCT patterns were compared using the Kruskal-Wallis test. Differences in serum KL-6 levels and in the extent of the opacities seen in the chest HRCT patterns were evaluated by the Mann-Whitney U test. The correlation coefficients between serum KL-6 levels and the extents of the opacities were calculated using the Spearman's log rank test. Quantitative differences were tested by the $\chi^{2}$ test for goodness of fit or by the Fisher's exact probability test. A p value of $<0.05$ was considered significant.

\section{RESULTS}

\section{Interpretation of chest HRCT images}

The interobserver variability before reaching consensus agreement was expressed as a Kappa value which was 0.90 for the predominant patterns and 0.80 for the extent of the opacities, indicating good agreement between the observers.

\section{Chest HRCT patterns}

Table 1 shows the categories of causal drugs and the chest HRCT patterns of the associated pneumonitis. There were seven patients with DAD, 11 with CIP, eight with BOOP/EP, and four with HP patterns. Cytotoxic agents caused a DAD or
Table 1 Causal drugs and chest HRCT patterns of pneumonitis

\begin{tabular}{llllll}
\hline Drug & $\mathbf{n}$ & DAD & CIP & BOOP/EP & HP \\
\hline Cytotoxic agents $^{*}$ & 7 & 3 & 3 & 1 & - \\
NSAIDs† & 7 & 1 & 1 & 3 & 2 \\
Antibiotics $\ddagger$ & 8 & 1 & 4 & 2 & 1 \\
Others§ & 8 & 2 & 3 & 2 & 1 \\
Total & 30 & 7 & 11 & 8 & 4 \\
\hline
\end{tabular}

$D A D=$ diffuse alveolar damage; $C I P=$ chronic interstitial pneumonia; $\mathrm{BOOP}=$ bronchiolitis obliterans organising pneumonia;

$\mathrm{HP}=$ hypersensitivity pneumonitis; $\mathrm{EP}=$ eosinophilic pneumonia;

NSAIDs = non-steroidal anti-inflammatory drugs.

${ }^{*}$ Cytotoxic agents included peplomycin $(n=3)$, bleomycin $(n=1)$, tegafur/uracil $(n=2)$, and methotrexate $(n=1)$.

†NSAIDs included loxoprofen $(n=2)$, diclofenac $(n=2)$, acetaminophen $(n=2)$, and mesalazine $(n=1)$.

$\ddagger$ Antibiotics included panipenem/betamiprom $(n=2)$, minocycline $(n=3)$, clarithromycin $(n=1)$, cefepime $(n=1)$, and cefozopran $(n=1)$. §Other agents included gold salt $(n=2)$, carvedilol $(n=1)$, bisoprolol $(n=1)$, maprotiline $(n=1)$, naftopidil $(n=1)$,

decamethylcyclopentasiloxane $(n=1)$, and herbal Gosha-jinki-gan

$(n=1)$.

CIP pattern in six out of seven patients, while non-steroidal anti-inflammatory drugs caused BOOP/EP or HP patterns in five out of seven patients. The other drugs showed no particular relationship with the pattern of pneumonitis.

\section{Relationship between serum KL-6 levels and chest HRCT patterns}

Figure 1 shows the levels of KL-6 at the time of diagnosis according to the type of pneumonitis. Serum KL-6 was increased in 16 of the 30 patients $(53.3 \%)$. There were no significant differences in sex or age between the patients with and without increased KL-6 levels (data not shown). There were significant differences in the serum KL-6 levels associated with the four chest HRCT patterns $(p<0.0001)$. All 16 patients with increased circulating KL-6 levels had either a DAD or a CIP pattern. None of the patients with BOOP/EP or HP patterns had an increased KL-6 level. KL-6 levels were therefore significantly higher in patients with DAD and CIP than in those with BOOP/EP or HP. Moreover, KL-6 levels were significantly higher in patients with DAD than in those

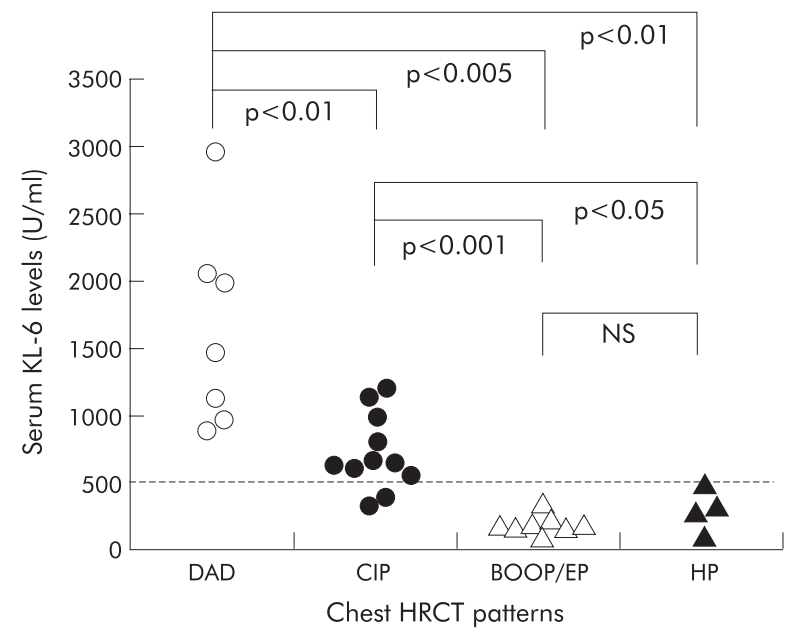

Figure 1 Relationship between serum levels of KL- 6 and patterns of pneumonitis. The horizontal dashed line represents the cut off level $(520 \mathrm{U} / \mathrm{ml})$ for KL-6. Serum levels of KL-6 were significantly increased in patients with DAD (open circle) or CIP (closed circle) compared with $\mathrm{BOOP} / \mathrm{EP}$ (open triangle) or HP (closed triangle) patterns. 
with CIP. However, there was no significant difference in the serum levels of KL-6 between patients with a BOOP/EP or HP pattern.

\section{Relationship between serum KL- 6 levels and extent of the opacities}

The extent of the opacities seen on the chest CT scan was significantly correlated with serum levels of KL-6 $\left(r_{\mathrm{s}}=0.525\right.$, $\mathrm{p}<0.01$ ), as shown in fig 2 . Since KL-6 was not increased in patients with a BOOP/EP or HP pattern, raised levels of KL-6 correlated primarily with the extent of DAD or CIP associated opacities. If BOOP/EP and HP patterns were excluded, there was still a significant correlation between KL-6 levels and extent of the opacities $\left(r_{\mathrm{s}}=0.569, \mathrm{p}=0.0245\right)$ which is comparable to that seen for all cases $\left(r_{\mathrm{s}}=0.525, \mathrm{p}<0.01\right)$. Disease extent was significantly higher in the DAD than in the CIP or BOOP/EP patterns $(\mathrm{p}<0.001)$. The CIP pattern also showed greater involvement of the opacities than did the BOOP/EP pattern $(p<0.01)$. However, the extent of the opacities seen in the HP pattern did not differ from those in the other groups.

\section{Serum KL-6 levels and clinical course}

Five out of seven patients with DAD, eight out of 11 with CIP, and all patients with a BOOP/EP or HP pattern showed clinical improvement which was defined as an improvement in both chest radiological findings and clinical laboratory examinations. The other five patients died from respiratory failure because of drug induced pneumonitis. Patients with increased levels of serum KL-6 at diagnosis had significantly higher mortality $(5 / 16 ; 31.3 \%)$ than the others $(0 / 14 ; 0 \%)$ $(p<0.05)$. Figure $3 A$ shows the changes in serum KL-6 levels in 11 survivors with DAD $(n=4)$ or CIP $(n=7)$ patterns. In 10 patients the serum KL-6 levels decreased significantly with response to withdrawal of the implicated drug and/or corticosteroid treatment. For one patient with a CIP pattern the serum KL-6 level at diagnosis was normal but increased after withdrawal of the implicated drug and then decreased with response to corticosteroid therapy. In contrast, serum levels of KL-6 either remained unchanged or increased in five non-survivors with a DAD $(n=2)$ or CIP $(n=3)$ pattern (fig 3B). In patients with DAD or CIP patterns, serial reduction in serum KL-6 levels was seen in 10 of 11 survivors

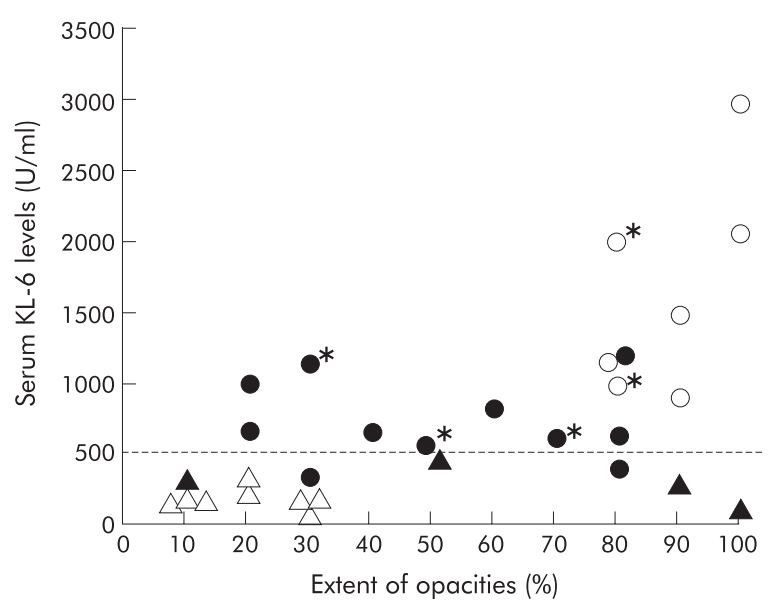

Figure 2 Correlation between serum KL-6 levels and extent of opacities. Serum levels of KL- 6 were significantly correlated with the extent of the opacities seen on chest HRCT scans $\left(r_{s}=0.525, \mathrm{p}<0.01\right)$. Each dot represents a patient with a DAD (open circle), CIP (closed circle), BOOP/EP (open triangle), or HP (closed triangle) pattern. *Nonsurvivors.
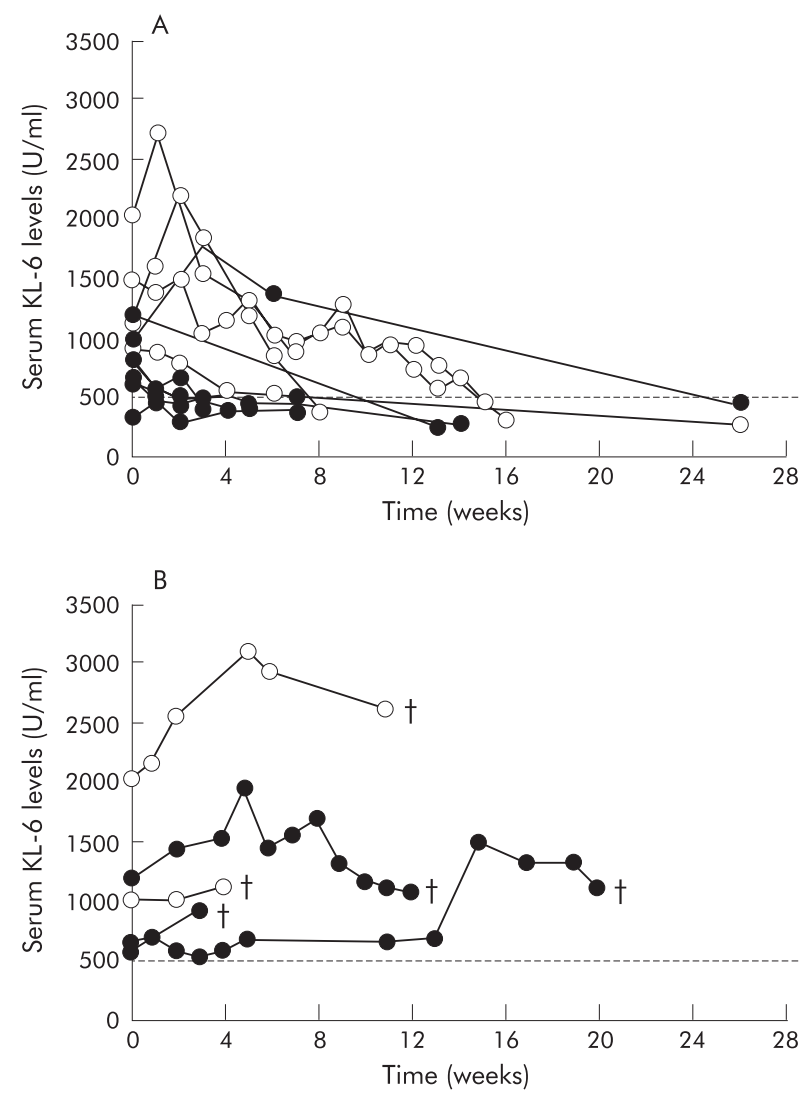

Figure 3 Changes in serum KL-6 levels in (A) 11 survivors and (B) five non-survivors with a DAD (open circle) or CIP (closed circle) pattern. The horizontal dashed line represents the cut off level $(520 \mathrm{U} / \mathrm{ml})$ for KL-6. Serum levels of KL-6 in survivors were significantly higher at diagnosis and decreased in response to withdrawal of the implicated drug and/or corticosteroid therapy. Serum levels of KL-6 in non-survivors either remained unchanged or increased without responding to treatment. †Death.

but in none of five non-survivors $(\mathrm{p}<0.001)$. There was no significant difference in disease extent on the chest CT scan between survivors and non-survivors in the DAD/CIP group. In all 10 patients with a BOOP/EP or HP pattern, serum levels of KL-6 remained below the cut off level throughout the clinical course (data not shown).

\section{DISCUSSION}

We have shown a relationship between the type of lung injury and serum levels of KL-6 in patients with drug induced lung disease. KL-6 was increased in particular types of lung injury such as those associated with a DAD or CIP pattern on the chest HRCT scan, but not in those with a BOOP/EP or HP pattern. The level of KL-6 increased or decreased in accordance with the clinical course of the disease in patients with a DAD or CIP pattern. A similar correlation has also been described in previous case reports. ${ }^{21-24}$ The overall sensitivity $(53.3 \%)$ of KL-6 for drug induced lung disease was obviously lower than that for other interstitial lung diseases such as idiopathic pulmonary fibrosis. ${ }^{13} 161720$ However, if we restrict our analysis to patients with a DAD or CIP pattern who comprised more than half the patients studied $(18 / 30)$, the sensitivity $(88.9 \%)$ is comparable to that seen for other interstitial lung diseases.

KL-6 levels did not increase in drug induced pneumonitis associated with a BOOP/EP or HP pattern. Similar to drug induced pneumonitis, serum KL-6 levels do not increase in 
patients with idiopathic BOOP or EP. BOOP/EP in the acute phase usually exhibited peribronchial or subpleural consolidation without fibrosis on the chest HRCT scan, similar to bacterial pneumonia. In many of these cases serum levels of KL-6 also remained within normal values. ${ }^{20} \mathrm{~A}$ high serum level of KL- 6 is one characteristic of the usual HP caused by inhalation of an organic antigen. ${ }^{13}{ }^{15}$ The appearance of the chest HRCT scan in drug induced pneumonitis with an HP pattern was essentially the same as that usually seen in the HP pattern associated with non-drug induced pneumonitis. However, the small centrilobular nodules typically seen in the more common form of HP were absent in drug induced pneumonitis with an HP pattern. The difference in KL-6 levels indicates that the mechanism may be substantially different between drug induced and the more common type of HP.

The primary cellular source of KL-6 is type II pneumocytes. ${ }^{13}$ The concentration of KL-6 is estimated to be extremely high in epithelial lining fluid. ${ }^{15}{ }^{17}$ An increase in circulating KL-6 levels in interstitial pneumonitis is therefore thought to be due to an increase in KL-6 production by regenerating alveolar type II pneumocytes and/or to an enhanced permeability following destruction of the air-blood barrier in the affected lungs. ${ }^{20}$ Serum levels of KL-6 were significantly increased in particular types of lung injury such as DAD and CIP, but not in injuries with a BOOP/EP or HP pattern. KL-6 levels were significantly correlated with the extent of the opacities detected by chest HRCT scanning in patients with drug induced lung diseases. KL-6 might be increased in these cases according to the extent of lung injury because patients with a DAD or CIP pattern had a significantly larger extent of opacities and significantly higher levels of serum KL-6 than did those with a BOOP/EP pattern. However, the HP pattern was not associated with an increased level of KL-6, even in cases where there was a large extent of opacities. Moreover, the CIP pattern was accompanied by increased serum levels of KL-6 that were independent of the extent of the opacities. Serum levels of KL-6 may therefore reflect not only the extent but also the type of lung injury involved.

In patients with DAD or CIP patterns, serial reduction in KL-6 levels was seen in 10 of 11 survivors but in none of five patients who died. We evaluated whether the five nonsurvivors had more extensive disease on CT scanning than the 11 survivors. However, there was no significant difference in disease extent on the chest CT scan between survivors and non-survivors in the DAD/CIP group. The predictive value of serial KL-6 measurement cannot therefore account for the differences in the disease extent on the CT scan.

In conclusion, serum KL-6 levels are increased in particular types of drug induced lung injury such as those associated with DAD and CIP patterns. It should be noted that DAD and CIP patterns are the most common patterns encountered in drug induced pneumonitis. ${ }^{8-10}$ Moreover, these types of pneumonitis are related to high morbidity and mortality. ${ }^{6}$ One limitation of the present study was the small number of patients included. Further prospective studies will be needed to determine whether measurement of KL-6 levels would be beneficial in the monitoring and early detection of pneumonitis in patients treated with various drugs. These studies should investigate drugs such as antineoplastic agents which can produce pneumonitis with a DAD or CIP pattern.

\section{ACKNOWLEDGEMENTS}

The authors thank Drs K Kondo, H Katayama, R Ito, and T Kadowaki for their technical assistance and for their help in the preparation of the manuscript.

\section{Authors' affiliations}

H Ohnishi, A Yokoyama, A Watanabe, H Hamada, J Higaki, Second Department of Internal Medicine, Ehime University School of Medicine, Ehime, Japan

Y Yasuhara, J Ikezoe, Department of Radiology, Ehime University School of Medicine, Ehime, Japan

T Naka, Department of Molecular Medicine, Osaka University Graduate School of Medicine, Osaka, Japan

M Abe, K Nishimura, Department of Respiratory Medicine, Ehime National Hospital, Ehime, Japan

N Kohno, Second Department of Internal Medicine and Department of Molecular and Internal Medicine, Graduate School of Biomedical Sciences, Hiroshima University, Hiroshima, Japan

\section{REFERENCES}

1 Cooper JA Jr, White DA, Matthay RA. Drug-induced pulmonary disease. Part 1: Cytotoxic drugs. Am Rev Respir Dis 1986;133:321-40.

2 Cooper JA Jr, White DA, Matthay RA. Drug-induced pulmonary disease. Part 2: Noncytotoxic drugs. Am Rev Respir Dis 1986;133:488-505.

3 Israel-Biet D, Labrune S, Huchon GJ. Drug-induced lung disease: 1990 review. Eur Respir J 1991;4:465-78.

4 Rosenow EC 3rd, Myers JL, Swensen SJ, et al. Drug-induced pulmonary disease. An update. Chest 1992;102:239-50.

5 Foucher $\mathrm{P}$, Biour $\mathrm{M}$, Blayac JP, et al. Drugs that may injure the respiratory system. Eur Respir J 1997;10:265-79.

6 Fraser RS, Müller NL, Colman N, et al. Drugs. In: Fraser RS, Müller NL, Colman N, eds. Fraser and Paré's diagnosis of diseases of the chest, 4th ed. Philadelphia: WB Saunders, 1999:2537-83.

7 Myers JL. Pathology of drug-induced lung disease. In: Katzenstein AL, Askin FB, eds. Katzenstein and Askin's surgical pathology of non-neoplastic lung disease, 4th ed. Philadelphia: WB Saunders, 1997:82-111.

8 Padley SPG, Adler B, Hansell DM, et al. High-resolution computed tomography of drug-induced lung disease. Clin Radiol 1992;46:232-6.

9 Rossi SE, Erasmus JJ, McAdams HP, et al. Pulmonary drug toxicity: radiologic and pathologic manifestations. Radiographics 2000;20:1245-59.

10 Webb WR, Müller NL, Naidich DP. Diseases characterized primarily by linear and reticular opacities. In: Webb WR, Müller NL, Naidich DP, eds. Highresolution CT of the lung, 3rd ed. Philadelphia: Lippincott Williams \& Wilkins, 2001:193-257

11 Kohno N, Akiyama M, Kyoizumi S, et al. Detection of soluble tumorassociated antigens in sera and effusions using novel monoclonal antibodies, $\mathrm{KL}-3$ and $\mathrm{KL}-6$, against lung adenocarcinoma. Jpn J Clin Oncol 1988; 18:203-16.

12 Kohno N, Inove $\mathrm{Y}$, Hamada $\mathrm{H}$, et al. Difference in sero-diagnostic values among KL-6-associated mucins classified as cluster 9. Int J Cancer Suppl 1994:8:81-3.

13 Kohno N, Kyoizumi S, Awaya $Y$, et al. New serum indicator of interstitial pneumonitis activity. Sialylated carbohydrate antigen KL-6. Chest 1989;96:68-73

14 Kohno N, Hamada H, Fujioka S, et al. Circulating antigen KL-6 and lactate dehydrogenase for monitoring irradiated patients with lung cancer. Chest 1992;102:117-22.

15 Kohno N, Awaya Y, Oyama T, et al. KL-6, a mucin-like glycoprotein, in bronchoalveolar lavage fluid from patients with interstitial lung disease. Am Rev Respir Dis 1993; 148:637-42.

16 Kohno N, Yokoyama A, Hirasawa Y et al. Comparative studies of circulating $\mathrm{KL}-6$, type III procollagen $\mathrm{N}$-terminal peptide and type IV collagen $7 \mathrm{~S}$ in patients with interstitial pneumonitis and alveolar pneumonia. Respir Med 1997;91:558-61

17 Takahashi T, Munakata M, Suzuki I, et al. Serum and bronchoalveolar fluid KL-6 levels in patients with pulmonary alveolar proteinosis. Am J Respir Crit Care Med 1998;158:1294-8.

18 Yokoyama A, Kohno N, Hamada H, et al. Circulating KL-6 predicts the outcome of rapidly progressive idiopathic pulmonary fibrosis. Am J Respir Crit Care Med 1998;158:1680-4.

19 Hamada H, Kohno N, Yokoyama A, et al. KL-6 as a serologic indicator of Pneumocystis carinii pneumonia in immunocompromised hosts. Intern Med 1998;37:307-10

20 Ohnishi H, Yokoyama A, Kondo K, et al. Comparative study of KL-6, surfactant protein-A, surfactant protein-D, and monocyte chemoattractant protein-1 as serum markers for interstitial lung diseases. Am J Respir Crit Care Med 2002;165:378-81.

21 Nakajima M, Manabe T, Mitekura H, et al. Levels of serum KL-6 in a patient with drug-induced pneumonitis. Nihon Kyobu Shikkan Gakkai Zasshi 1997:35:813-7, (in Japanese).

22 Endoh Y, Hanai R, Uto K, et al. KL-6 as a potential new marker for amiodarone-induced pulmonary toxicity. Am J Cardiol 2000;86:229-31.

23 Matsumoto T, Nakamura K, Sugisaki K, et al. Misoprostol-induced pneumonitis. Nihon Kokyuki Gakkai Zasshi 2000;38:447-51, (in Japanese).

24 Miyata M, Sakuma F, Fukaya E, et al. Detection and monitoring of methotrexate-associated lung injury using serum markers KL-6 and SP-D in rheumatoid arthritis. Intern Med 2002;41:467-73.

25 Naranjo CA, Busto U, Sellers EM, et al. A method for estimating the probability of adverse drug reactions. Clin Pharmacol Ther 1981:30:239-45.

26 Obuchowski NA, Zepp RC. Simple steps for improving multiple-reader studies in radiology. AJR Am J Roentgenol 1996;166:517-21. 